\title{
Early-Stage Hodgkin Lymphoma: A Case-Based Review of the Recent Literature
}

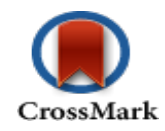

Dennis L. Cooper *

Section of Hematologic Malignancies, Yale Cancer Center, New Haven, CT

*Corresponding Author: Cooper, D. L., 140 Patten Road, North Haven, USA. Tel: 203-376-0051; E-mail: dennislcooper64@ gmail.com

\begin{abstract}
The treatment of early-stage Hodgkin lymphoma remains controversial with combined modality therapy and chemotherapy alone representing acceptable options. Observation studies show a progressive decline in the use of radiation secondary to late complications but also suggest a decrease in survival for non-irradiated patients. Early PET CT scans have been incorporated into strategies of response-adapted therapy but have been imperfect in identifying patients who may require more aggressive treatment. In the absence of radiation, further improvement in the cure rate is likely to require better chemotherapy such as the incorporation of two cycles of dose-escalated BEACOPP or possibly the substitution of brentuximab for bleomycin. The treatment of patients who have relapse after abbreviated chemotherapy alone is not standardized and it remains unclear whether these patients should all receive highdose chemotherapy and autologous stem cell rescue or whether a significant fraction can be salvaged with conventional chemotherapy and radiation. Great care is required to ensure that patients considered for abbreviated treatment fulfil the eligibility requirements for inclusion as there are significant differences in the distinction between favorable and unfavorable in the most influential cooperative group studies.
\end{abstract}

\section{Case Presentation}

A 42-yr-old woman presented with left neck and axillary adenopathy and a lymph biopsy confirmed nodular sclerosing Hodgkin lymphoma. She did not have B symptoms and a PET CT scan showed disease in the left neck and axilla as well as the mediastinum. There were no areas of bulky $(>10 \mathrm{~cm})$ disease and the mediastinal involvement was less than one-third of the thoracic diameter. She had a normal CBC with an ESR of $30 \mathrm{~mm} / \mathrm{H}$. Chemistries including serum albumin were unremarkable. She was staged as IIA.

How should this patient be treated?

\section{Introduction}

Despite the high likelihood of prolonged survival and cure for more than $85 \%$ of patients after first line therapy for patients with early-stage Hodgkin lymphoma, ${ }^{[1-5]}$ the choice of treatment remains controversial. Currently, the two major treatment strategies for favorable early stage Hodgkin lymphoma are combined modality thera-
Received Date: September 01, 2015

Accepted Date: September 09, 2015

Published Date: September 12, 2015

Citation: Cooper, D. L. Early-Stage Hodgkin Lymphoma: A Case-Based Review of the Recent Literature (2015) Int J Hematol Ther 1(1): 1-6.

DOI: $10.15436 / 2381-1404.15 .006$

py (CMT) with abbreviated chemotherapy followed by consolidative low-dose (20-30 Gy) radiation (involved field or involved nodes) or chemotherapy alone for a variable number of cycles ${ }^{[1,2,4-7]}$. Large database reviews, such as by Olszewski et al. show that there has been a progressive decline in the use of CMT with approximately $45 \%$ of patients receiving CMT in 2011 compared to $59 \%$ in $2003^{[8]}$. Of some concern is that these studies also show a decreased survival when chemotherapy is used alone, a finding also reached by a Cochrane meta-analysis of 5 randomized clinical trials that compared CMT and chemotherapy alone $^{[9]}$.

\section{Chemotherapy Alone}

Not withstanding the limitations of observation studies ${ }^{[10]}$ and the meta-analysis above, the question remains as to how effective is the use of chemotherapy, specifically abbreviated ABVD when used 
without radiation in early stage HL? A large multi-institutional study with long follow-up was performed by the NCI-Canada $\left(\mathrm{NCIC} \mathrm{H6)}{ }^{[6]}\right.$. In that study, favorable patients (Table 1), were randomized to ABVD alone for 4-6 cycles versus subtotal nodal irradiation (STNI) without chemotherapy. The unfavorable group, which would have included our patient (age $>40$ ), was randomized to ABVD x 4-6 cycles versus 2 cycles of ABVD plus STNI. The number of treatment cycles in the chemotherapy alone groups was based on the results of an interim CT scan performed after the second cycle of therapy (PET CT not available at this time) with patients achieving $\mathrm{CR}$ or $\mathrm{CRu}(39 \%)$ treated for 2 additional cycles (4 total) and those with $<\mathrm{CRu}(61 \%)$ treated for 4 more cycles ( 6 total). CMT in the unfavorable patients was associated with superior freedom from progression (FFP) compared to chemotherapy alone. Interestingly, CMT in the unfavorable group also resulted in a better FFP than chemotherapy alone in the favorable group, supporting a non-cross resistant activity for radiation. However, this study is most well known for showing a better overall survival in patients treated with chemotherapy alone due to an increase in solid tumors (as well as an unusual increase in probably unrelated mortal events) in those who received CMT.

Table 1: Ineligibility and Adverse Risk Factors in Recent Early-Stage

\begin{tabular}{|c|c|c|c|c|}
\hline & NCIC H6 & $\begin{array}{c}\text { GHSG H10, } \\
\text { H11 }\end{array}$ & $\begin{array}{c}\text { EORTC/LYSA/ } \\
\text { FIL H10F and } \\
\text { H10U }\end{array}$ & $\begin{array}{c}\text { UK } \\
\text { RAPID }\end{array}$ \\
\hline $\begin{array}{l}\text { Ineli- } \\
\text { gibil- } \\
\text { ity }\end{array}$ & $\begin{array}{c}\text { B symptoms } \\
\text { Bulky Med } \\
\geq 1 / 3 \mathrm{TD}) \\
\text { Abdominal } \\
\text { disease (Pel- } \\
\text { vic disease } \\
\text { allowed) }\end{array}$ & $\begin{array}{c}\text { IIB }+ \text { Bulky } \\
\text { Med }(\geq 1 / 3 \text { TD } \\
\text { IIB plus } \\
\text { extranodal } \\
\text { disease }\end{array}$ & $\begin{array}{l}\text { Subdiaphragm } \\
\text { disease }\end{array}$ & $\begin{array}{c}\text { B symp- } \\
\text { toms } \\
\text { Bulky } \\
\text { Med } \geq \\
1 / 3 \text { TD) } \\
\text { Infradia- } \\
\text { phragm }\end{array}$ \\
\hline $\begin{array}{l}\text { Risk } \\
\text { Fac- } \\
\text { tors }\end{array}$ & $\begin{array}{l}\text { Age } \geq 40 \\
\geq 4 \text { sites } \\
\text { of disease } \\
E S R \geq 50 \\
\text { LD or MC } \\
\text { histology }\end{array}$ & $\begin{array}{c}\geq 3 \text { areas of } \\
\text { disease } \\
\text { Med } \geq 1 / 3 \text { TD } \\
\text { B symptoms } \\
\text { ESR } \geq 50 \\
\text { without B } \\
\text { symptoms, } \\
\text { B symptoms } \\
\text { plus ESR } \geq 30\end{array}$ & $\begin{array}{c}\text { Age }>50 \\
\geq 4 \text { areas of } \\
\text { disease } \\
\text { Med } \geq 1 / 3 \text { TD } \\
\mathrm{A}+\mathrm{ESR} \geq 50 \\
\mathrm{~B}+\mathrm{ESR} \geq 30\end{array}$ & \\
\hline
\end{tabular}

B symptoms and bulky mediastinal disease excluded patients from early stage protocols on the NCIC and UK RAPID trials. Patients with risk factors are treated on different protocol for unfavorable patients. The NCIC, GHSG and EORTC/LYSA/FIL cooperative groups have separate criteria for risk factors. Per the GHSG studies, ipsilateral neck/ supraclavicular/infraclavicular, mediastinal/right/left hilar, ipsilateral inguinal/femoral are each considered one area.

LD: lymphocyte depleted, MC: mixed cellularity, TD: thoracic diameter

Although at the time of publication, it was readily acknowledged that STNI was obsolete and more carcinogenic than modern involved field treatment fields and doses ${ }^{[11,12]}$, it seems likely that the results from this study further fueled the drive to eliminate radiation entirely from treatment. However, it should be noted that the $86 \%$ FFP in the unfavorable group treated with chemotherapy alone ${ }^{[6]}$ probably represents a "best case" result as patients with bulky mediastinal disease and B symptoms were excluded from the NCIC H6 study. Thus, in a randomized study from Sloan Kettering that included unfavorable (including a small percentage of stage IIIA), subjects, the FFP after six cycles of ABVD was $81 \%{ }^{[13]}$. Therefore, the expected FFP from ABVD alone in a broader group of early-stage patients are probably at best, around $80-85 \%$.

\section{Interim Pet Ct Scans \\ Role In Modulating Therapy}

The early response to therapy as measured on PET CT scan has been shown to be a powerful predictor of outcome in patients with advanced disease as patients achieving a negative scan after 2 cycles of ABVD have an excellent prognosis while those with residual FDG-avid disease have a dismal disease-free and overall survival ${ }^{[14]}$. These observations have stimulated clinical trials of "response-adapted" treatment in which therapy is de-escalated in good responders and potentially intensified in those with persistent FDG uptake after 2-3 cycles of $\mathrm{ABVD}^{[15]}$. In early stage HL, two recent trials of PET response-adapted therapy have shown promising but imperfect results in identifying patients who may require more intensive treatment. The EORTC/LYSA/FIL trial tested whether involved nodal radiation therapy (INRT) could be omitted in patients with stage I-II supradiaphragmatic disease who achieved a negative PET CT scan after two cycles of $\mathrm{ABVD}^{[5]}$. Favorable patients who achieved a negative scan in the experimental group were treated with 2 additional cycles of ABVD alone whereas in the experimental unfavorable group, those who achieved negative interim scans were treated with 4 additional (total of six) cycles of ABVD. Patients in the standard arms received one or two cycles of ABVD followed by involved node radiation. However, an interim analysis of non-inferiority of the chemotherapy alone arms showed an excess of events in the chemotherapy alone groups and the study was stopped. Nevertheless, because the relapse rate was still very low and the overall survival was excellent, the authors ambiguously concluded that the correct treatment strategy was "in the eye of the beholder".

In the RAPID trial from the United Kingdom, which excluded patients with B symptoms and bulky mediastinal disease, PET CT scans were performed after 3 cycles of ABVD ${ }^{[4]}$. Patients with negative scans were randomized to IFRT or no further therapy. This study also showed a lower PFS (90.7) at 3 years in the non-irradiated group, a number that was slightly but significantly worse than in those who received IFRT as assigned $(97 \%)$. The authors of the latter study, while acknowledging the slightly higher recurrence rate in the patients who did not receive radiation, recommended that chemotherapy alone should be considered in patients who achieve a negative PET CT scan. These two studies have established chemotherapy without radiation as acceptable treatment strategies in early stage patients who achieve negative interim PET CT scans with the caveat that relapses were slightly more frequent in the non-irradiated patients but not often enough to justify exposing all patients to radiation. The number of chemotherapy cycles was different in the EORTC/LYSA/FIL study ${ }^{[4-6]}$ and RAPID study ${ }^{[3]}$ as were the disease characteristics of the patients and the timing of the interim PET CT scan (Table 2). 
Table 2: Current Treatment for Early Stage Hodgkin Lymphoma

\begin{tabular}{|c|c|c|c|c|}
\hline \multicolumn{2}{|c|}{ CMT } & \multicolumn{3}{|c|}{ CHEMO ALONE } \\
\hline \multirow{2}{*}{$\begin{array}{c}\text { Favorable } \\
\text { GHSG H10 }\end{array}$} & \multirow{2}{*}{$\begin{array}{c}\text { Unfavorable* } \\
\text { GHSG H11 }\end{array}$} & \multicolumn{2}{|c|}{ EORTC/LYSA/FIL } & \multirow{2}{*}{$\begin{array}{l}\text { RAP- } \\
\text { ID }^{* * *}\end{array}$} \\
\hline & & Favorable & Unfavorable $^{* *}$ & \\
\hline $\begin{array}{c}\text { ABVD x } 2 \\
+20 \mathrm{~Gy} \\
\text { IFRT }\end{array}$ & $\begin{array}{l}\text { ABVD x } 4 \\
+30 \text { Gy }\end{array}$ & ABVD $\times 2$ & ABVD $\times 2$ & $\begin{array}{l}\text { ABVD } \\
\times 3\end{array}$ \\
\hline \multirow{2}{*}{\multicolumn{2}{|c|}{$\begin{array}{l}\text { The GHSG trials did not } \\
\text { include PET CT but current } \\
\text { trials include PET CT after } \\
2 \text { cycles with treatment } \\
\text { adapted to response }\end{array}$}} & PET Neg\# & PET Neg" & $\begin{array}{c}\text { PET } \\
\mathrm{Neg}^{\# \#}\end{array}$ \\
\hline & & ABVD $\times 2$ & ABVD $\times 4$ & $\begin{array}{l}\text { No } \\
\text { further } \\
\mathrm{Rx}\end{array}$ \\
\hline
\end{tabular}

* GHSG: Unfavorable: $\geq 3$ areas of disease, bulky mediastinal $(\geq$ $1 / 3$ thoracic diameter, ESR $\geq 50$ without $\mathrm{B}$ symptoms or $\geq 30$ with $\mathrm{B}$ symptoms, extranodal extension

** EORTC/LYSA/FIL: Unfavorable: Age $>50, \geq 4$ nodal areas, bulky mediastinal $(\geq 1 / 3$ thoracic diameter, $\mathrm{ESR} \geq 50$ without symptoms or $\geq$ 30 with B symptoms

\# In the EORTC/LYSA/FIL study, a positive PET scan resulted in treatment intensification with dose-escalated BEACOPP and 30 Gy involved field radiation

*** RAPID: Excludes B symptoms and bulky mediastinal disease \#\# In the RAPID study, a positive PET CT resulted in 1 additional cycle of ABVD and 30 Gy RT

It is important to note that in both of the risk-adapted studies described above, the interim PET CT scan underwent a contextual or situational interpretation (Table 3). In other words, because a negative result of the interim PET CT scan resulted in a de-escalation of treatment (no radiation), the equivalent of a Deavuville score of 3 (uptake equal or greater than mediastinum but less than liver) rather than the generally accepted score of 4 (uptake greater than liver), was considered positive in order to reduce the number of false negative scans and to avoid under-treatment. This is almost certainly the reason why only $75 \%$ of patients in the RAPID trial had a negative scan after 3 cycles of ABVD, a value about 8 points lower than that achieved in patients with advanced disease after one less cycle of $\mathrm{ABVD}^{[14]}$. From a practical point of view, this requires careful collaboration with the nuclear medicine radiologist including the generation of a Deauville score.

Table 3: Contextual Interpretation of Interim FDG-PET

\begin{tabular}{|l|l|}
\hline Score & PET \\
\hline 1 & NO UPTAKE ABOVE BASELINE: \\
\hline 2 & UPTAKE $\leq$ MEDIASTINUM \\
\hline 3 & UPTAKE $>$ MEDIASTINUM BUT $\leq$ LIVER \\
\hline 4 & UPTAKE MODERATELY > LIVER \\
\hline 5 & UPTAKE MARKEDLY > LIVER \\
\hline
\end{tabular}

In the Deauville grading system, a score of 1-3 is generally considered to be negative for pathologic uptake. However, in clinical trials in which therapy is going to be de-escalated for good early responders, such as withholding radiation, a score of 3 is considered positive in order to avoid under treatment. Conversely, if therapy were going to be intensified because of a positive study, such as changing to a more aggressive chemotherapy regimen or a higher dose of radiation, a score of 4 is used in order to reduce the chances of over-treatment.
It should also be emphasized that based on the entry criteria and treatment in the two large response-adapted, cooperative group trials described above, neither study above has shown that a negative interim PET CT allows an abbreviated chemotherapy regimen without radiation in patients with bulky mediastinal disease or B symptoms. Thus, in the RAPID trial, patients with bulky mediastinal disease or B symptoms were excluded and in the EORTC/LYSA/FIL unfavorable arms (including bulky mediastinal disease, B symptoms or elevated ESR without B symptoms) patients were treated with six cycles of ABVD even after an early negative PET CT.

\section{Treatment Of Patients With Persistently Positive Pet Ct Scans After 2-3 Cycles Of Abvd}

As described above, in the EORTC/LYSA/FIL and RAPID trials, the incidence of disease progression in patients who achieved a negative interim PET CT was approximately 5\% and $10 \%$, respectively, consistent with an excellent prognosis. The incidence of positive scans was $20 \%$ in the EORTC/LYSA/ FIL and $25 \%$ in the RAPID trial. The reason for the lower incidence of positive scans in the EORTC/LYSA/FIL compared to the RAPID trial is uncertain and somewhat disconcerting as the opposite distribution would have been expected. Thus, the patient population of the EORTC/LYSA/FIL included patients with bulky mediastinal disease and B symptoms whereas the latter patients were excluded from the RAPID trial. In addition, the earlier PET CT (after 2 cycles of ABVD in the EORTC/LYSA/ FIL compared with 3 cycles in the RAPID trial) would have intuitively been expected to be associated with a higher percentage of positive scans at the earlier time point. Although both studies incorporated a central reading, the difference in the percentage of positive studies raises a question about the similarity and reproducibility of the PET CT interpretation, particularly as these strategies are exported into clinical practice.

Interestingly, only about $10 \%$ of patients with a positive scan in the RAPID trial experienced disease progression (similar to the patients with negative scans) indicating either that a positive interim scan has a very poor positive predictive value (nearly $2 / 3$ of patients with a "positive" scan had a Deauville score of 3 ) or that the subsequent (standard) treatment with an additional cycle of ABVD and 30 Gy IFRT was effective in sterilizing residual disease.

In patients with a positive interim study on the EORTC/ LYSA/FIL trial, therapy on the experimental arms was intensified to two cycles of dose-escalated BEACOPP followed by 30 Gy INRT. Patients with a positive interim scan on the standard arms received ABVD plus 30 Gy INRT. Preliminary results suggested a strong benefit for augmented therapy after an interim positive scan with an increase in 5-year PFS from 77\% to $91 \%$ and an increase in 5 year OS from $89 \%$ to $96 \%$ (Presented by Raemaekers et al. at ICML, Lugano June 2015).

\section{Where Do We Stand On The Risk-Adapted Approach To The Treatment Of Early Stage Disease?}

Adjusting treatment based on a contextual interpretation of the interim PET CT has not been deployed outside the confines of a few clinical trials in which a centralized review was performed. The fact that patients with advanced disease who achieve a Deauville score of 3 are scored as negative while patients with early-stage disease are scored as positive encap- 
sulates the potential complexity and confusion surrounding the interpretation of the interim scan. Similarly, while there is consensus that a persistently positive scan requires additional treatment including radiation, it remains unclear that patients with a Deauville score 3 (which comprised $>60 \%$ of the patients on the RAPID trial and an uncertain percentage on the EORTC/ LYSA/FIL trial) should be treated as aggressively as patients with a score of 4-5 as the latter score is more consistent with disease that is grossly resistant to ABVD and that likely requires an intensification of therapy (e.g., dose-escalated BEACOPP). Therefore, outside of a clinical trial, it is not clear risk-adapted therapy with PET CT is ready for generalized use.

\section{Combined Modality Therapy(CMT)}

Until recently, the unchallenged standard of care for patients with early-stage disease, has been CMT. If our patient is treated with CMT, how many courses of chemotherapy and what dose of radiation should she receive? The paradigm-changing GHSG H10 trial is one of the largest and most important studies of CMT in early-stage $\mathrm{HL}^{[2]}$. In that study, it was found that 2 cycles of ABVD and 20 Gy radiation were as effective as 4 cycles of ABVD and $30 \mathrm{~Gy}$, thus establishing the former as the new standard in patients with favorable (no risk factors) early-stage disease. Risk factors that pushed patients into the companion GHSG H11 trial for unfavorable disease (Table 1) included large mediastinal mass ( $\geq$ one-third of the maximum thoracic diameter, extranodal disease, involvement of three or more nodal areas and elevation of the ESR ( $\geq 50 \mathrm{~mm}$ without $\mathrm{B}$ symptoms and $\geq 30 \mathrm{~mm} / \mathrm{h}$ for stage IB or IIB). As our patient has three lymph node areas ( $\mathrm{L}$ neck, L axilla and mediastinal/hilar) she would not have been eligible for the H10 trial but would have been included in the GHSG H11 trial for unfavorable patients.

At this juncture, it is important to note that the definition of favorable and unfavorable early stage disease is different in the large cooperative groups (Table 1). For example, only $110 / 181$ patients treated with chemotherapy alone on the NCIC H6 trial would have been candidates for the GHSG H10 (favorable) trial; in a retrospective study comparing the NCIC H6 and GSHD early stage trials, 3 or more nodal areas of disease was the most common reason for failing to qualify for treatment on the GHSG H10 study ${ }^{[16]}$.

As our patient would have been a candidate for the GHSG H11 trial, she would have been randomized to four cycles of either ABVD or baseline doses of BEACOPP and then received 20 Gy or 30 Gy IFRT $^{[1]}$. This study showed that ABVD plus 30 Gy was as effective but less toxic than baseline BEACOPP and 30 Gy but that ABVD plus 20 Gy of RT was probably inferior to ABVD plus $30 \mathrm{~Gy}$. Accordingly, four cycles of ABVD plus 30 Gy of RT is considered the standard for this group of patients and by extension, would be considered appropriate for all patients with early stage, unfavorable disease. As the latter treatment clearly represents additional cycles of chemotherapy and a higher dose of radiation than for favorable disease, it is essential that a patient's disease characteristics be carefully scrutinized before recommending the very appealing program of two cycles of ABVD plus 20 Gy RT.

\section{Improving Cure Rates}

In the GHSG H11 trial for unfavorable patients, freedom from progression remained $<90 \%$ and per the authors con- clusion, a less than optimal result. As an earlier EORTC/GELA study did not show that 6 cycles of ABVD plus IFRT was superior to 4 cycles plus IFRT ${ }^{[17]}$, the latter program likely represents the best that can be expected from ABVD plus IFRT. Thus, the ability to further reduce and possibly eliminate radiation while improving cure rates with initial treatment is likely to require more effective chemotherapy. Although baseline doses of BEACOPP were not better than ABVD in both the GHSG H11 trial and the EORTC/GELA H9U trials ${ }^{[1,17]}$, the GHSG H14 trial showed that two cycles of dose-escalated BEACOPP followed by 2 cycles of ABVD (“2 + 2") was superior to four cycles of ABVD (all patients received 30 Gy radiation) ${ }^{[7]}$. The ongoing GHSG H17 trial for unfavorable early-stage disease is testing whether radiation can be deleted in patients who achieve an interim negative PET CT after the second cycle of the $2+2$ regimen with the hypothesis that the deeper remission resulting from this more intensive and effective program will create a better platform for a radiation-free treatment than ABVD.

The $2+2$ regimen was clearly associated with more acute toxicity than ABVD but thus far, there has not been an increase in secondary cancers or myelodysplasia ${ }^{[7]}$. Importantly, while return of menses was generally delayed in women treated with $2+2$ compared to ABVD, more than $90 \%$ of women had a normal menstrual cycle after treatment and there was no decrease in the percentage of women who had successful pregnancies. In men, spermatogenesis was more acutely impacted by $2+2$ than ABVD x 4 ; however in patients who survived $\geq 4$ years, there were no differences in serum inhibin or FSH levels, consistent with recovery of spermatogenesis ${ }^{[18]}$.

In North America, it seems likely that the reluctance to use dose-escalated BEACOPP will likely extend to patients with early stage patients. As a result, perhaps the greatest hope for improving outcome in the future is by substituting brentuximab for bleomycin in a "new ABVD" where the B is brentuximab. By virtue of the remarkable single agent activity in patients with relapsed disease, including some unmaintained prolonged complete remissions ${ }^{[19]}$, brentuximab is arguably the most active single drug in the treatment of HL and almost certainly more active than bleomycin, a drug whose contribution to ABVD remains uncertain $^{[20]} 40$ years after the regimen was first introduced ${ }^{[21]}$.

Thus far, a preliminary study of ABrenVD in early stage unfavorable Hodgkin lymphoma (but also including several patients with more advanced disease) showed that $14 / 15$ patients achieved a negative PET CT scan after 2 cycles including 10/10 with bulky mediastinal disease ${ }^{[22]}$. Although 30 Gy adjuvant radiation was given without any signal thus far for pulmonary toxicity, it is expected that if other ongoing trials confirm the superiority of the brentuximab-containing regimen, trials with reduced or no RT will undoubtedly be tested.

\section{Treatment Of Relapsed Disease}

Based on the recently published results of the RAPID trial, it seems likely that a greater number of patients with early stage disease will be treated with abbreviated chemotherapy alone. It is therefore important to note that treatment for recurrent disease in the latter setting has not been well studied and is certainly not standardized. Some investigators have recommended salvage chemotherapy followed by high dose therapy and autologous transplant ${ }^{[23]}$, similar to other patients who have relapsed after chemotherapy or CMT. Nevertheless, while this 
approach seems appropriate for patients who relapse after CMT or after 4-6 cycles of ABVD, it seems likely that since many of the relapsing patients relapsed because they did not receive radiation (particularly relapses in previous sites of disease), conventional salvage treatment including radiation could be considered.

In the RAPID study discussed above, 22 patients relapsed after abbreviated chemotherapy alone; 2 of 4 patients treated with salvage chemotherapy alone died. However, all of the remaining patients treated with radiation alone ${ }^{[5]}$, chemotherapy plus $\mathrm{RT}^{[6]}$ or high dose chemotherapy plus autologous stem cell rescue remain alive. These data, albeit in a small number of patients, raise the question as to whether patients who achieve a negative PET CT with salvage chemotherapy, can be treated with consolidative radiation rather than high-dose therapy and autologous stem cell rescue ${ }^{[24]}$.

\section{Summary}

Currently, there are two acceptable approaches to the treatment of patients with early Hodgkin lymphoma (Table 2). CMT regimens have offered a slightly higher initial cure rate but with an uncertain risk for late complications despite decreases in radiation treatment field and doses. Response-adapted chemotherapy based on interim PET CT during treatment is an attractive strategy for reducing the burden of therapy in a substantial percentage of patients while maintaining cure rates. However, despite using an altered (lower) threshold for interpreting an interim scan as positive, relapse rates in patients treated with chemotherapy alone remain slightly higher than after CMT, consistent with the non-cross resistant activity of radiation. Conversely, there is not yet a consensus on the best strategy for treating patients with an interim positive scan although it seems likely that more intensive therapy will be required for the very small percentage of patients with high FDG activity on interim PET CT. It is likely that further improvements in chemotherapy such as the inclusion of two cycles of dose-escalated BEACOPP or possibly the incorporation of brentuximab, may further reduce the need for radiation except in poor responders or in the relapsed setting. From a practical point of view, the different definitions of favorable and unfavorable risk factors in the different cooperative groups make it imperative to accurately assess whether patients are appropriate for the treatment that is considered.

\section{References}

1. Eich, H. T., Diehl, V., Gorgen, H., et al. Intensified chemotherapy and dose-reduced involved-field radiotherapy in patients with early unfavorable Hodgkin's lymphoma: final analysis of the German Hodgkin Study Group HD11 trial. (2010) J Clin Oncol 28(27): 4199-4206.

2. Engert, A., Plutschow, A., Eich, H. T., et al. Reduced treatment intensity in patients with early-stage Hodgkin's lymphoma. (2010) N Engl J Med 363(7): 640-652.

3. Ferme, C., Eghbali, H., Meerwaldt, J. H., et al. Chemotherapy plus involved-field radiation in early-stage Hodgkin's disease. (2007) N Engl J Med 357(19): 1916-1927.

4. Radford, J., Illidge, T., Counsell, N., et al. Results of a trial of PET-directed therapy for early-stage Hodgkin's lymphoma. (2015) N Engl J Med 372: 1598-1607.
5. Raemaekers, J. M., Andre, M. P., Federico, M., et al. Omitting radiotherapy in early positron emission tomography-negative stage I/II Hodgkin lymphoma is associated with an increased risk of early relapse: Clinical results of the preplanned interim analysis of the randomized EORTC/LYSA/FIL H10 trial. (2014) JCO 32: 1188-1194.

6. Meyer, R.M., Gospodarowicz, M. K., Connors, J. M., et al. ABVD alone versus radiation-based therapy in limited-stage Hodgkin's lymphoma. (2012) N Engl J Med 366: 399-408.

7. Von Tresckow, B., Plutschow, A., Fuchs, M., et al. Dose-intensification in early unfavorable Hodgkin's lymphoma: final analysis of the German Hodgkin Study Group HD14 trial. (2012) J Clin Oncol 30(9): 907-913.

8. Olszewski, A. J., Shrestha, R., Castillo, J. J. Treatment selection and outcomes in early-stage classical hodgkin lymphoma: analysis of the national cancer data base. (2015) J Clin Oncol. 33(6): 625-633.

9. Herbst, C., Rehan, F. A., Brillant, C., et al. Combined modality treatment improves tumor control and overall survival in patients with early stage Hodgkin's lymphoma: a systematic review. (2010) Haematologica 95(3): 494-500.

10. Levine, M. N., Julian, J.A. Registries that show efficacy: good, but not good enough. (2008) J Clin Oncol 26(33): 53165319 .

11. De Bruin, M. L., Sparidans, J., van't Veer, M. B., et al. Breast cancer risk in female survivors of Hodgkin's lymphoma: lower risk after smaller radiation volumes. (2009) J Clin Oncol 27(26): 4239-4246.

12. Hodgson, D. C., Koh, E. S., Tran, T.H., et al. Individualized estimates of second cancer risks after contemporary radiation therapy for Hodgkin lymphoma. (2007) Cancer 110(11): 25762586.

13. Straus, D. J., Portlock, C. S., Qin, J., et al. Results of a prospective randomized clinical trial of doxorubicin, bleomycin, vinblastine, and dacarbazine (ABVD) followed by radiation therapy (RT) versus ABVD alone for stages I, II, and IIIA nonbulky Hodgkin disease. (2004) Blood 104(12): 3483-3489.

14. Gallamini, A., Polliack, A. Interim and end-treatment positron emission tomography scan in aggressive B-cell lymphoma: we still lack an interpretation key. (2014) Leuk Lymphoma 55(7): 1447-1448.

15. Gallamini, A., Patti, C., Viviani, S., et al. Early chemotherapy intensification with BEACOPP in advanced-stage Hodgkin lymphoma patients with a interim-PET positive after two ABVD courses. (2011) Br J Haematol. 152(5): 551-560.

16. Hay, A. E., Klimm, B., Chen, B. E., et al. An individual patient-data comparison of combined modality therapy and ABVD alone for patients with limited-stage Hodgkin lymphoma. (2013) Ann Oncol 24(12): 3065-3069.

17. Ferme, C., Divine, M., Vranovsky, A., et al. Four ABVD and Involved-Field Radiotherapy in Unfavorable Supradiaphragmatic Clinical stages (CS) I-II Hodgkin's Lymphoma (HL): Preliminary Results of the EORTC-GELA H9-U Trial (abstract). (2005) Blood 106: 813.

18. Behringer, K., Mueller, H., Goergen, H., et al. Gonadal function and fertility in survivors after Hodgkin lymphoma treatment within the German Hodgkin Study Group HD13 to HD15 trials. (2013) J Clin Oncol 31(2): 231-239.

19. Gopal, A. K., Chen, R., Smith, S. E., et al. Durable remissions in a pivotal phase 2 study of brentuximab vedotin in re- 
lapsed or refractory Hodgkin lymphoma. (2015) Blood 125(8): 1236-1243.

20. Canellos, G. P., Duggan, D., Johnson, J., et al. How important is bleomycin in the adriamycin + bleomycin + vinblastine + dacarbazine regimen? (2004) J Clin Oncol 22(8): 1532-1533. 21. Bonadonna, G., Zucali, R., Monfardini, S., et al. Combination chemotherapy of Hodgkin's disease with adriamycin, bleomycin, vinblastine, and imidazole carboxamide versus MOPP. (1975) Cancer 36(1): 252-259.

22. Kumar, A., Yahalom, J., Shoder, H., et al. Safety and Early Efficacy in an Ongoing Pilot Study of Brentuximab Vedotin and AVD Chemotherapy Followed by 30 Gray Involved-Site Radio- therapy for Newly Diagnosed, Early Stage, Unfavorable Risk Hodgkin Lymphoma (abstract). (2014) Blood 124: 3085.

23. Connors, J. M. Chemotherapy only in early stage Hodgkin lymphoma: more relapses but the same survival--what to do? (2014) Curr Hematol Malig Rep 9(3): 217-221.

24. Moskowitz, A. J., Schoder, H., Yahalom, J., et al. PET-adapted sequential salvage therapy with brentuximab vedotin followed by augmented ifosamide, carboplatin, and etoposide for patients with relapsed and refractory Hodgkin's lymphoma: a non-randomised, open-label, single-centre, phase 2 study. (2015) Lancet Oncol 16(3): 284-292. 